\title{
PHENOLIC PRESERVATIVE REMOVAL FROM COMMERCIAL INSULIN FORMULATIONS REDUCES TISSUE INFLAMMATION, WHILE MAINTAINING EUGLYCEMIA
}

Adam Mulka', Brianne E. Lewis', Li Mao', Roshanak Sharafieh², Shereen Kesserwan ${ }^{1}$, Rong Wu ${ }^{2}$, Donald L. Kreutzer ${ }^{2}$, Ulrike Klueh ${ }^{1 *}$

\author{
1Department of Biomedical Engineering, \\ Integrative Biosciences Center \\ Wayne State University, Detroit, MI \\ ${ }^{2}$ Department of Surgery, \\ School of Medicine. \\ University of Connecticut, \\ Farmington, CT. \\ ${ }^{*}$ Corresponding Author: \\ Ulrike Klueh Ph. D. \\ Department of Biomedical Engineering, \\ Wayne State University \\ Detroit, MI 48202 \\ Email: klueh@wayne.edu
}

Table of Contents

Supplementary Figures 1 \& 2................ -2

Supplementary Table 1.............................

Supplementary Table 2............................. -4 


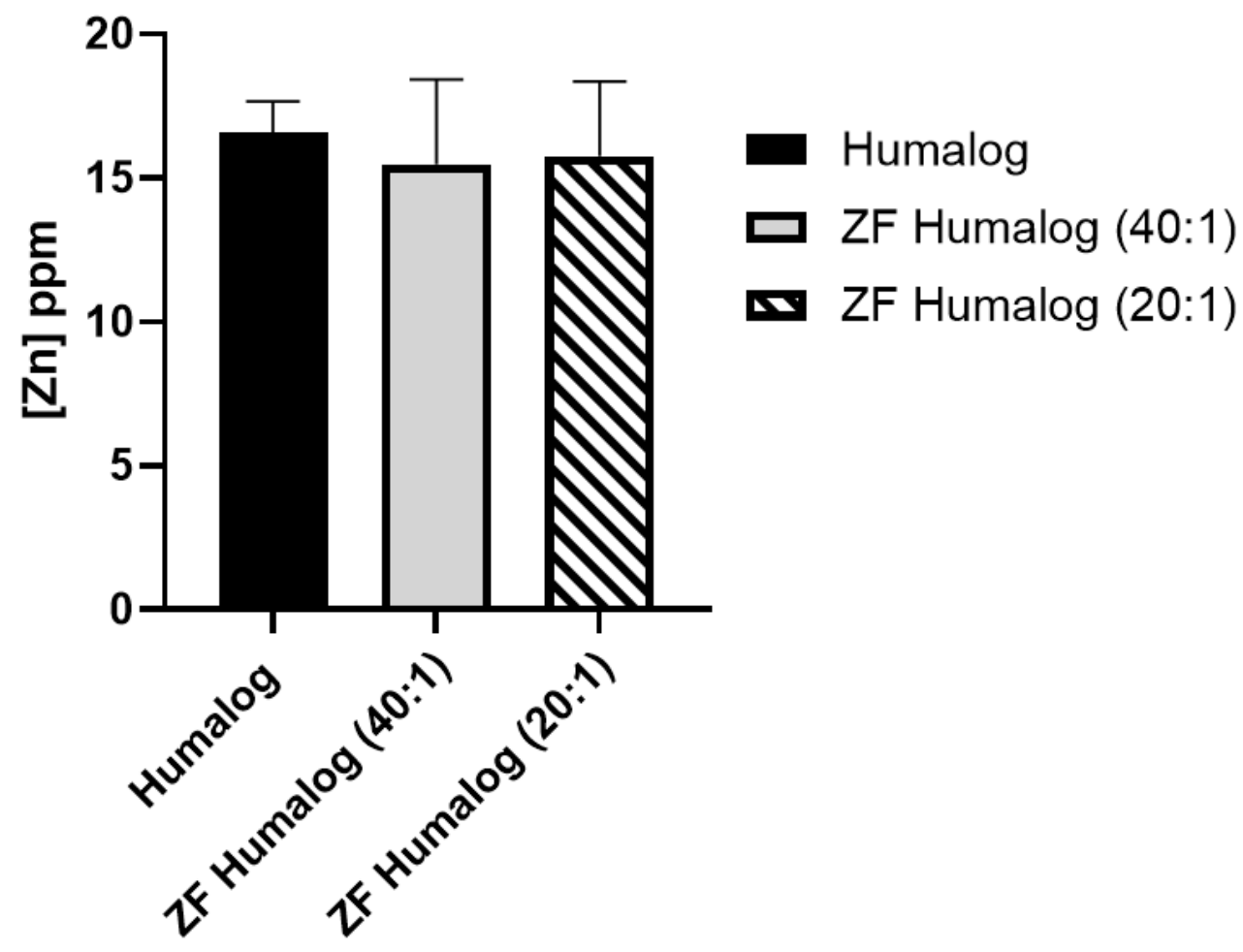

Figure S-1: ICP-MS for Zinc Filtration: Zeolite $Y$ filtered Humalog samples were submitted to the Lumigen core facility for ICP-MS analysis. Unfiltered Humalog used as a control. This data demonstrates the selective exclusion of phenol and $\mathrm{m}$-cresol by the zeolite.

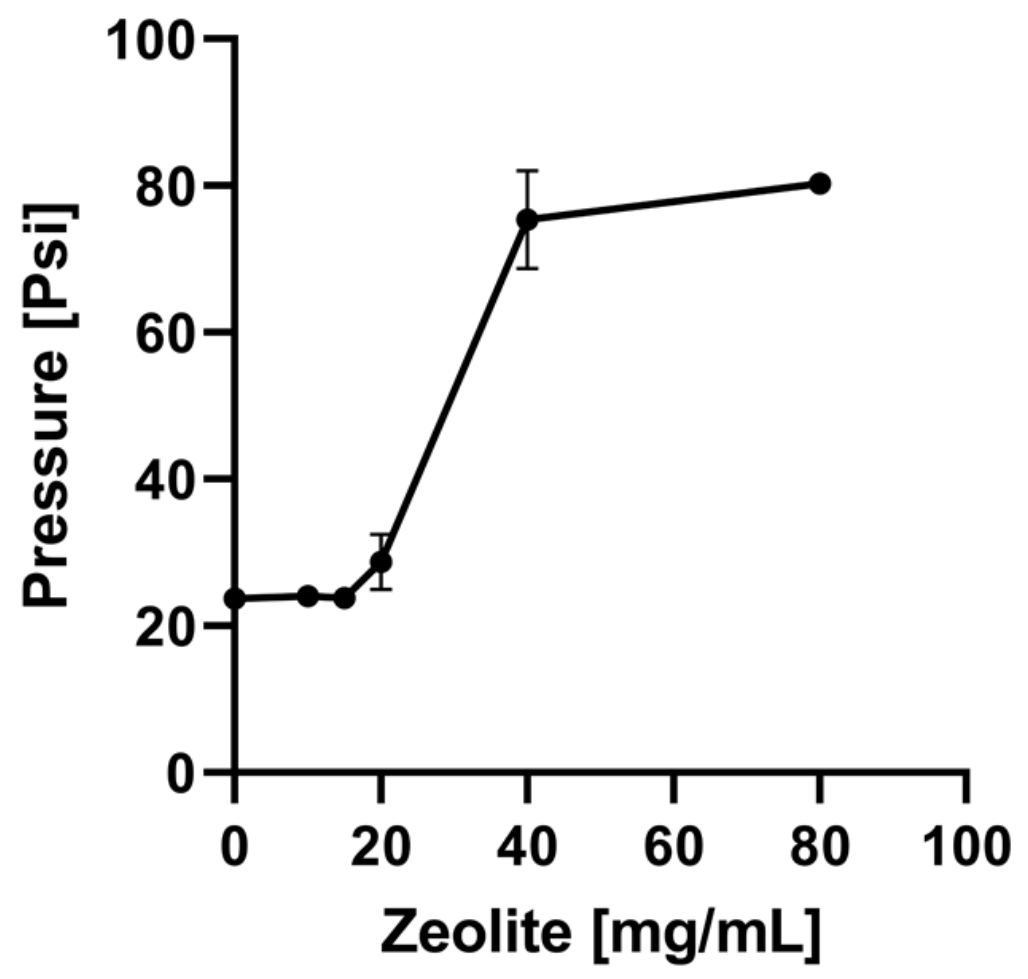

Figure S-2: In-line Pressure Monitoring: As the amount of Zeolite $Y$ in the in-line filter system increased, in-line backpressure increased proportionally. This data demonstrates the need to minimize zeolite/ alleviate pressure in the proposed system. 


\begin{tabular}{|c|c|c|c|c|c|c|c|c|c|}
\hline \multicolumn{2}{|l|}{ Cell Type } & \multirow{2}{*}{\multicolumn{2}{|c|}{ RAW Macrophage }} & \multirow{2}{*}{\multicolumn{2}{|c|}{ BMMC }} & \multirow{2}{*}{\multicolumn{2}{|c|}{ 3T3-L1 Fibroblast }} & \multirow{2}{*}{\multicolumn{2}{|c|}{$\begin{array}{l}\text { 3T3-L1 } \\
\text { Fat Cell }\end{array}$}} \\
\hline \multirow{2}{*}{$\begin{array}{l}\text { Dilution } \\
\text { Ratio }\end{array}$} & \multirow[t]{2}{*}{ Treatment } & & & & & & & & \\
\hline & & 1-day & 3-day & 1-day & 3-day & 1-day & 3-day & 1-day & 3-day \\
\hline \multirow[t]{5}{*}{$1 / 3$} & I vs. D & NS & NS & NS & NS & $<0.04$ & NS & NS & $<0.0001$ \\
\hline & I vs. S & $<0.0001$ & $<0.0001$ & $<0.0001$ & $<0.0001$ & $<0.0001$ & $<0.0001$ & $<0.0001$ & $<0.0001$ \\
\hline & I vs. ZI & $<0.0001$ & $<0.0001$ & $<0.0001$ & $<0.0001$ & $<0.0001$ & $<0.0001$ & $<0.0001$ & $<0.0001$ \\
\hline & D vs. $S$ & $<0.0001$ & $<0.0001$ & $<0.0001$ & $<0.0001$ & $<0.0001$ & $<0.0001$ & $<0.0001$ & $<0.0001$ \\
\hline & D vs. ZD & $<0.0001$ & $<0.0001$ & $<0.0001$ & $<0.0001$ & $<0.0001$ & $<0.0001$ & $<0.0001$ & $<0.0001$ \\
\hline \multirow[t]{5}{*}{$1 / 6$} & I vs. D & $<0.0001$ & $<0.0001$ & $<0.0001$ & 0.0002 & $<0.0001$ & $<0.0001$ & $<0.0001$ & $<0.0001$ \\
\hline & I vs. S & $<0.0001$ & $<0.0001$ & $<0.0001$ & $<0.0001$ & $<0.0001$ & $<0.0001$ & $<0.0001$ & $<0.0001$ \\
\hline & I vs. ZI & $<0.0001$ & $<0.0001$ & $<0.0001$ & $<0.0001$ & $<0.0001$ & $<0.0001$ & $<0.0001$ & $<0.0001$ \\
\hline & D vs. $S$ & $<0.0001$ & $<0.0001$ & $<0.0001$ & $<0.0001$ & NS & $<0.0001$ & $<0.0001$ & NS \\
\hline & D vs. ZD & $<0.0001$ & $<0.0001$ & $<0.0001$ & $<0.0001$ & $<0.0001$ & $<0.0001$ & $<0.0001$ & NS \\
\hline \multirow[t]{5}{*}{$1 / 12$} & I vs. D & $<0.0001$ & $<0.0001$ & $<0.01$ & $<0.01$ & NS & $<0.0001$ & 0.0136 & NS \\
\hline & I vs. S & $<0.0001$ & $<0.0001$ & $<0.0001$ & $<0.0001$ & NS & $<0.0001$ & $<0.0001$ & $<0.0001$ \\
\hline & I vs. ZI & $<0.0001$ & $<0.0001$ & $<0.0001$ & $<0.0001$ & NS & $<0.0001$ & NS & $<0.0001$ \\
\hline & D vs. $S$ & $<0.0001$ & $<0.0001$ & $<0.0001$ & $<0.0001$ & NS & $<0.0001$ & $<0.0001$ & $<0.001$ \\
\hline & D vs. ZD & $<0.0001$ & $<0.0001$ & $<0.0001$ & $<0.0001$ & 0.02 & $<0.0001$ & NS & 0.03 \\
\hline \multirow[t]{5}{*}{$1 / 24$} & I vs. D & $<0.0001$ & $<0.0001$ & $<0.01$ & $<0.01$ & NS & $<0.01$ & $<0.001$ & NS \\
\hline & I vs. S & $<0.0001$ & $<0.0001$ & $<0.0001$ & NS & NS & $<0.0001$ & $<0.0001$ & $<0.0001$ \\
\hline & I vs. ZI & $<0.0001$ & $<0.0001$ & $<0.0001$ & NS & NS & NS & 0.0029 & $<0.0001$ \\
\hline & D vs. $S$ & NS & $<0.0001$ & $<0.0001$ & 0.01 & NS & $<0.0001$ & NS & $<0.0001$ \\
\hline & D vs. ZD & NS & $<0.0001$ & NS & NS & NS & NS & NS & NS \\
\hline \multirow[t]{5}{*}{$1 / 48$} & I vs. D & NS & $<0.01$ & 0.001 & $<0.0001$ & 0.01 & 0.01 & NS & NS \\
\hline & I vs. S & NS & $<0.0001$ & NS & $<0.0001$ & NS & $<0.0001$ & $<0.0001$ & NS \\
\hline & I vs. ZI & NS & $<0.0001$ & $<0.0001$ & $<0.0001$ & NS & $<0.0001$ & NS & $<0.02$ \\
\hline & D vs. $S$ & NS & 0.0021 & $<0.0001$ & NS & NS & $<0.0001$ & NS & $<0.01$ \\
\hline & D vs. ZD & NS & NS & NS & NS & NS & $<0.0001$ & NS & NS \\
\hline \multirow[t]{5}{*}{$1 / 96$} & I vs. D & NS & NS & $<0.001$ & NS & NS & NS & NS & NS \\
\hline & I vs. S & NS & NS & $<0.0001$ & NS & $<0.0001$ & NS & NS & 0.02 \\
\hline & I vs. ZI & NS & NS & $<0.0001$ & 0.02 & 0.02 & $<0.0001$ & $<0.01$ & $<0.0001$ \\
\hline & D vs. $S$ & NS & NS & NS & NS & $<0.0001$ & NS & NS & 0.003 \\
\hline & D vs. ZD & $<0.001$ & NS & 0.001 & $<0.0001$ & $<0.0001$ & NS & NS & NS \\
\hline \multirow[t]{5}{*}{$1 / 192$} & I vs. D & $<0.01$ & NS & NS & NS & 0.0014 & NS & $<0.01$ & $<0.0001$ \\
\hline & I vs. S & NS & <0.0001 & $<0.01$ & 0.02 & $<0.0001$ & NS & $<0.01$ & $<0.0001$ \\
\hline & I vs. ZI & $<0.001$ & NS & NS & $<0.0001$ & NS & NS & NS & $<0.0001$ \\
\hline & D vs. $S$ & $<0.01$ & $<0.0001$ & $<0.0001$ & 0.04 & $<0.01$ & NS & NS & NS \\
\hline & D vs. ZD & $<0.001$ & NS & $<0.0001$ & $<0.0001$ & NS & NS & $<0.001$ & NS \\
\hline
\end{tabular}

Table S-1: In vitro Mouse cell Statistics: Statistics calculated using two-way Anova $(p<0.05)$ for 1 - and 3-days of exposure in mouse RAW Macrophages, 3T3-L1 Fibroblast, 3T3-L1 derived Fat cells, and BMMC. Tukey multiple comparisons made between saline (S), diluent (D), insulin (I), Zeolite filtered insulin (ZI), and Zeolite filtered diluent (ZD). 


\begin{tabular}{|c|c|c|c|c|c|c|c|}
\hline \multicolumn{2}{|c|}{ Cell Type } & \multicolumn{2}{|c|}{ PBMC } & \multicolumn{2}{|c|}{ HMC-1 } & \multicolumn{2}{|c|}{ THP-1 } \\
\hline \multirow{2}{*}{$\begin{array}{l}\text { Dilution } \\
\text { Ratio }\end{array}$} & \multirow{2}{*}{ Treatment } & & & & & & \\
\hline & & 1-day & 3-day & 1-day & 3-day & 1-day & 3-day \\
\hline \multirow[t]{5}{*}{$1 / 3$} & I vs. D & NS & NS & NS & NS & NS & NS \\
\hline & I vs. S & $<0.0001$ & $<0.0001$ & $<0.0001$ & $<0.0001$ & $<0.0001$ & $<0.0001$ \\
\hline & I vs. ZI & $<0.0001$ & $<0.0001$ & $<0.0001$ & $<0.0001$ & $<0.0001$ & $<0.0001$ \\
\hline & D vs. $S$ & $<0.0001$ & $<0.0001$ & $<0.0001$ & $<0.0001$ & $<0.0001$ & $<0.0001$ \\
\hline & D vs. ZD & $<0.0001$ & $<0.0001$ & $<0.0001$ & $<0.0001$ & $<0.0001$ & $<0.0001$ \\
\hline \multirow[t]{5}{*}{$1 / 6$} & I vs. D & $<0.0001$ & $<0.0001$ & 0.02 & $<0.01$ & $<0.01$ & NS \\
\hline & I vs. S & $<0.0001$ & $<0.0001$ & $<0.0001$ & $<0.0001$ & $<0.0001$ & $<0.0001$ \\
\hline & I vs. ZI & $<0.0001$ & $<0.0001$ & $<0.0001$ & $<0.0001$ & $<0.0001$ & $<0.0001$ \\
\hline & D vs. $S$ & $<0.0001$ & $<0.0001$ & $<0.0001$ & $<0.0001$ & $<0.0001$ & $<0.0001$ \\
\hline & D vs. ZD & $<0.0001$ & $<0.0001$ & $<0.0001$ & $<0.0001$ & $<0.0001$ & $<0.0001$ \\
\hline \multirow[t]{5}{*}{$1 / 12$} & I vs. D & NS & $<0.0001$ & NS & 0.03 & $<0.01$ & $<0.0001$ \\
\hline & I vs. S & NS & $<0.0001$ & $<0.0001$ & $<0.0001$ & $<0.0001$ & $<0.0001$ \\
\hline & I vs. ZI & $<0.001$ & $<0.0001$ & $<0.0001$ & $<0.0001$ & $<0.0001$ & $<0.0001$ \\
\hline & D vs. $S$ & NS & $<0.0001$ & $<0.0001$ & $<0.0001$ & $<0.0001$ & NS \\
\hline & D vs. ZD & 0.0001 & $<0.0001$ & $<0.0001$ & $<0.0001$ & $<0.0001$ & $<0.0001$ \\
\hline \multirow[t]{5}{*}{$1 / 24$} & I vs. D & NS & $<0.0001$ & NS & NS & $<0.0001$ & $<0.0001$ \\
\hline & I vs. S & NS & $<0.0001$ & $<0.0001$ & $<0.0001$ & $<0.0001$ & $<0.0001$ \\
\hline & I vs. ZI & NS & 0.0002 & $<0.0001$ & NS & $<0.0001$ & $<0.0001$ \\
\hline & D vs. S & NS & NS & $<0.0001$ & $<0.01$ & $<0.0001$ & $\mid 0.0304$ \\
\hline & D vs. ZD & $<0.0001$ & NS & $<0.0001$ & $<0.0001$ & $<0.0001$ & $<0.0001$ \\
\hline \multirow[t]{5}{*}{$1 / 48$} & I vs. D & NS & $<0.0001$ & NS & NS & $<0.0001$ & $<0.0001$ \\
\hline & I vs. S & NS & $<0.0001$ & $<0.0001$ & $<0.01$ & $<0.0001$ & $<0.0001$ \\
\hline & I vs. ZI & $<0.0001$ & $<0.001$ & $<0.0001$ & NS & $<0.0001$ & $<0.0001$ \\
\hline & D vs. S & NS & 0.04 & $<0.0001$ & 0.03 & 0.02 & NS \\
\hline & D vs. ZD & NS & $<0.0001$ & 0.0015 & 0.02 & NS & NS \\
\hline \multirow{5}{*}{$1 / 96$} & I vs. D & $<0.0001$ & $<0.0001$ & 0.0126 & NS & $<0.001$ & $<0.0001$ \\
\hline & I vs. S & NS & $<0.0001$ & $<0.0001$ & NS & $<0.0001$ & NS \\
\hline & I vs. ZI & NS & NS & 0.0051 & NS & $<0.0001$ & $<0.0001$ \\
\hline & D vs. $S$ & NS & NS & $<0.0001$ & NS & $<0.01$ & $<0.0001$ \\
\hline & D vs. ZD & $<0.01$ & $<0.01$ & NS & 0.02 & $<0.01$ & NS \\
\hline \multirow[t]{5}{*}{$1 / 192$} & I vs. D & NS & $<0.0001$ & 0.001 & $<0.0001$ & NS & NS \\
\hline & I vs. S & NS & $<0.0001$ & $<0.0001$ & NS & $<0.0001$ & 0.0008 \\
\hline & I vs. ZI & NS & $<0.0001$ & 0.003 & NS & $<0.0001$ & $<0.0001$ \\
\hline & D vs. $S$ & $<0.03$ & $>0.9999$ & $<0.0001$ & NS & $<0.0001$ & 0.0408 \\
\hline & D vs. ZD & $<0.0001$ & $<0.0001$ & 0.0001 & $<0.0001$ & $<0.0001$ & $<0.0001$ \\
\hline
\end{tabular}

Table S-2: In vitro Human cell Statistics: Statistics calculated using two-way Anova $(p<0.05)$ for 1 - and 3-days of exposure in human PBMC, HMC-1, and THP-1 cells. Tukey multiple comparisons made between saline (S), diluent (D), insulin (I), Zeolite filtered insulin (ZI), and Zeolite filtered diluent (ZD). 